\title{
PERINATAL OUTCOME IN SEVERE PREECLAMPSIA PATIENTS IN RELATION TO ALTERED LFT
}

Manohar Tankasali, Manjula Patil

1. Assistant Professor. Department of Obstetrics \& Gynaecology, S. N. Medical College and HSK hospital \& Research Centre, Bagalkot.

2. Assistant Professor. Department of Anatomy, S. N. Medical College and HSK hospital \& Research Centre, Bagalkot.

\section{CORRESPONDING AUTHOR:}

Dr. Manohar Tankasali,

Assistant Professor,

Department Of OBG,

S. N Medical College And HSK hospital and Research Centre, Bagalkot.

E-mail: drmanohartankasali123@gmail.com

INTRODUCTION: Preeclampsia is one of the most common causes of perinatal morbidity and mortality in both developed and underdeveloped countries. Severe preeclampsia (SPE) can have catastrophic effects if treatment is delayed. Liver is involved in most of the cases of preeclampsia which manifests initially with alterations in the liver function test (LFT) which can be used as an early indicator of the complications in SPE patients. AIMS AND OBJECTIVES: To study the perinatal outcome in severe preeclampsia patients in relation to LFT. MATERIAL AND METHODS: All women with diastolic pressure $>110 \mathrm{~mm}$ of $\mathrm{Hg}$ were included in the study considering the exclusion criteria. Patient followed up for the perinatal outcome. RESULTS: Perinatal outcome with relation to LFT are as follows: (overall incidence in all pre-eclampsia cases, $48 \%$ of total study cases had altered LFT values. Numbers of preterm cases were $52 \%$ (Vs $43 \%$ ), which was the most common foetal complication. Apgar of 1 min and 5 min with $<7$ were $59.5 \%$ (Vs. 45\%). NICU admission was necessary in $62.5 \%$ (Vs.5 52\%) showing $2 / 3$ of neonatal need special neonatal observation and management. Foetal outcome were. IUGR- 54\% (Vs. 48\%) Hypoxia - 41.6\% (Vs 32\%) MAS - 14.6\% (Vs. 10\%) ARDS - 21.8\% (Vs 15\%). CONCLUSION: Detection of increased LFT in cases of SPE is a special risk category, associated with increased rate of perinatal complications, compared to SPE with normal LFT. Such cases need special attention with early detection and referral to higher centre with better facilities of ICU, laboratories and NICU set up to reduce the complications and mortality.

INTRODUCTION: Hypertensive disorders complicating pregnancies are common and form one of the deadly triad along with the hemorrhage and infection that contributes greatly to maternal and fetal morbidity and mortality. Among the hypertensive disorders preeclampsia (PE) is one of the most common causes of perinatal morbidity and mortality resulting in estimated 35-300 deaths per 1000 births depending on neonatal support capabilities of the hospital delivering care. This mortality rate is almost double that of normotensive pregnancies ${ }^{1,2}$.

Preeclampsia (PE) is a pregnancy specific disorder that usually occurs after 20 weeks of gestation. It can be defined as blood pressure of $140 \mathrm{~mm}$ of $\mathrm{Hg}$ systolic or higher or $90 \mathrm{~mm}$ of $\mathrm{Hg}$ diastolic or higher that occurs after 20 weeks of gestation in women with previously normal blood pressure along with proteinuria of $0.3 \mathrm{~g}$ or higher in $24 \mathrm{hrs}$ urine sample. (ACOG bulletin) Preeclampsia can be mild or severe. Severe preeclampsia is associated with significant maternal and neonatal morbidity and mortality. Severe preeclampsia (SPE) is defined as the presence of 
one or more of the following criteria: (a) blood pressure (BP) of $160 \mathrm{mmHg}$ or higher systolic or $110 \mathrm{mmHg}$ or higher diastolic on two occasions at least 6 hours apart while the patient is on bed rest; (b) proteinuria of $5 \mathrm{~g}$ or higher in a 24-hour urine specimen or $3+$ or greater on two random urine samples collected at least 4 hours apart; (c) oliguria of less than $500 \mathrm{~mL}$ in 24 hour with other associated signs and symptoms. ${ }^{3}$

Pre-eclampsia can manifest with few maternal symptoms and signs or as isolated intrauterine growth restriction (IUGR). Eclampsia may be the first presentation of the condition. If unrecognized, preeclampsia can progress to the syndrome of hemolysis, elevated liverenzyme levels and low platelet count (HELLP) and eclampsia. HELLP syndrome is noted in 5$10 \%$ of patients with preeclamptic symptoms. Mortality is $7-35 \%$ and perinatal mortality of the child may be up to $40 \% .{ }^{4}$ Pre-eclampsia and HELLP syndrome are far more dangerous to the fetus than to the mother with rates of IUGR and premature delivery and fetal and neonatal death rates of 6 to $37 \%$, deaths are likely the result of placental insufficiency and hypoxia. ${ }^{5}$

Liver is involved in at least $10 \%$ of women with pre eclampsia. ${ }^{6-10}$ Abnormal liver function tests occur in $20 \%$ to $30 \%$ of pregnancies complicated by pre-eclampsia and are associated with poor maternal and fetal outcomes.11,12 Liver involvement is more common in severe preeclampsia constituting the primary cause of death in $15-20 \%$ of fatal cases. 7,13

Many of the complications associated with preeclampsia are result of bad obstetric control, delay in diagnosis and late referral to the tertiary centre. ${ }^{2}$

As hepatic damage is commonly observed in preeclampsia patients, whether these alterations in LFT's can be utilized as an early indicator of complications of severe preeclampsia has to be tested for. Here is such an attempt to know the perinatal outcome in case of severe preeclampsia patients with altered LFT to see whether altered LFT can be utilized as an early indicator of complications of SPE, so that one can intervene and provide tertiary care to prevent the bad obstetric outcome.

AIM: To study the perinatal outcome in severe preeclampsia patients in relation to LFT.

MATERIAL AND METHODS: Material: The study cases were patients who got admitted for treatment of high BP during pregnancy in S. N. Medical College and HSK Hospital and Research Centre, Bagalkot during the year 2010 to 2012.

Method of collection: All pregnant women with period of gestation of 28-40 weeks recorded to have diastole BP of $110 \mathrm{mmHg}$ and above were considered in study group applying inclusion and exclusion criteria. Exclusion criteria: All patients with present or recent past history of liver disease, patients on hepatotoxic drugs.

Blood pressure measurement: Blood pressure was measured in right arm either sitting or lying position, measured preferably two times four hours apart of bed rest. Cut off point for diastole was korotkoff phase Vth sound.

Investigations: Blood sample was taken for LFT (AST \ALT \Total and Direct bilirubin $\backslash A G$ ratio \LDH \Alkaline phosphatase). Any one-parameter abnormality in LFT was considered as abnormal LFT. Guidelines of Sibai, for range of upper limits of normal LFT were considered. ${ }^{4}$

Treatment and follow up: Patients were treated for high blood pressure by using standard protocols. Patients were followed up until discharge. Foetal complications likePreterm/IUD/IUGR/Hypoxia/MAS/need for NICU admission etc were looked for.

Statistical methods applied: Frequencies and Contingency table analysis. All the statistical calculations were done through SPSS Version 14.0 for Windows. 


\section{RESULTS Graph 1. LFT in severe PE in different age group}

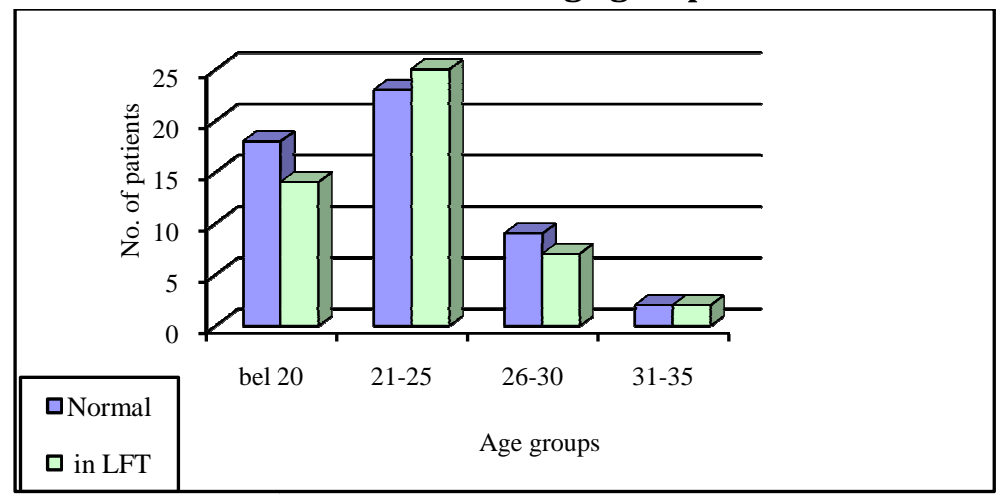

Most of patients were in the age group of 20- 25 year in the total study cases. SPE was more commonly seen in younger age group $(<25 \mathrm{Yr})$ 80\%. Among patients with raised LFT also maximum cases were seen in the same age group (81.3\%). Mean age group was 22.3 age.(CC = $.082: \mathrm{P}=0.879 \mathrm{NS}$ )

Graph. 2: Parity and LFT in severe PE:

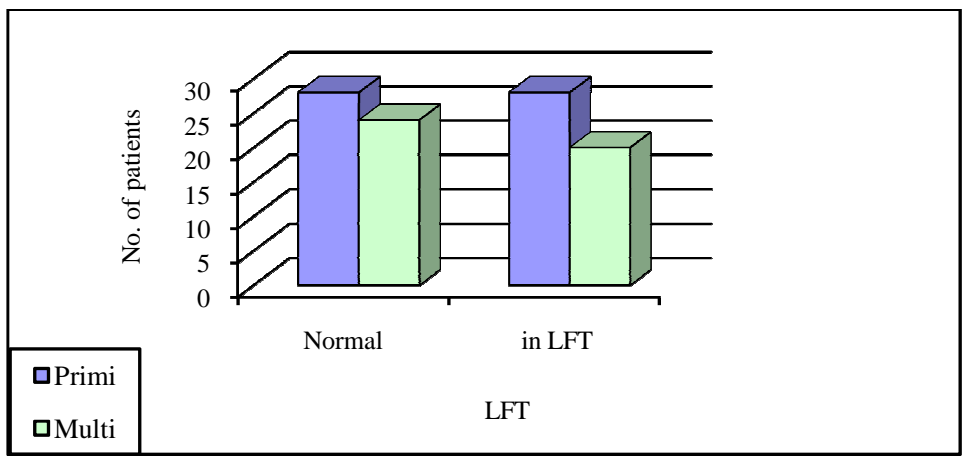

In overall study group number of primigravida patients were $56 \%$ and multigravida were $44 \%$. In patients with raised LFT primigravida were $58.3 \%$ and multigravida were $41.7 \%$. (CC=. 045; $\mathrm{P}=0.652 \mathrm{NS}$ )

\section{Graph 3. Booking status and LFT in SPE:}

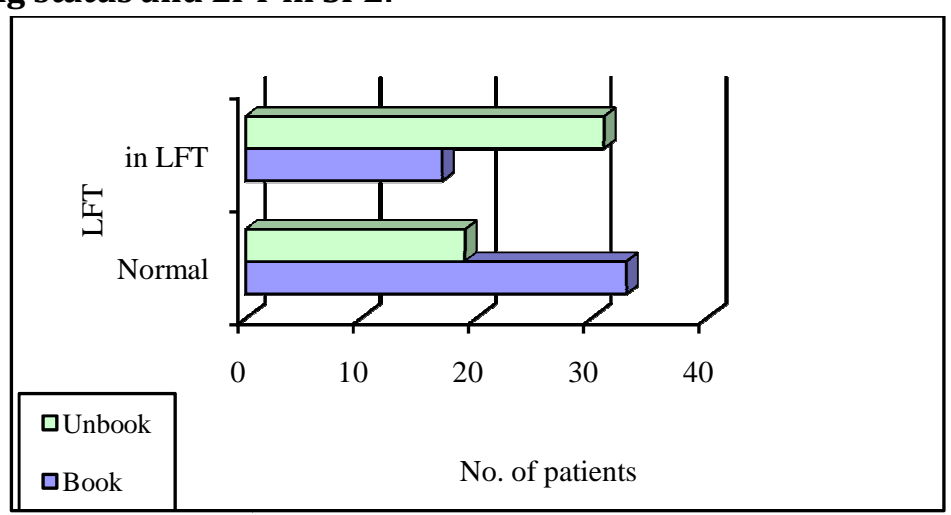

In overall study group number of booked and unbooked cases was equal. In patients with raised LFT unbooked cases were more (64.6\%) showing complications are more common in unbooked 
cases. Statistical significant relation is seen between raised LFT and unbooked cases. (CC $=0.270$; $\mathrm{P}=0.005$ Significant $)$

Table no. 1 Baby out comes at the time of discharge and LFT in severe PE:

\begin{tabular}{|l|l|l|l|}
\hline \multirow{2}{*}{ BAD } & LFT & Yes & Total \\
\cline { 2 - 4 } & No & 31 & 69 \\
\hline Alive & 38 & 11 & 22 \\
\hline IUD & 11 & 6 & 9 \\
\hline NM & 3 & 48 & 100 \\
\hline Total & 52 &
\end{tabular}

Out of total study cases, live babies at the time of discharge were 69 (69\%), deaths due to IUDs were 22 (22\%), and deaths in neonatal period were 9 (9\%). Among group with raised LFT live were $31(64.6 \%)$, deaths due to IUDs were $11(22.9 \%)$, and deaths in neonatal period were 6 (12.5\%). (CC=0.124; $\mathrm{P}=0.460)$.

Table no. 2 Apgar of 1 Min and LFT in severe PE:

\begin{tabular}{|l|lll|l|}
\hline AP 1 & LFT & & Total \\
\cline { 2 - 4 } & No & Yes & \\
\hline $0-3$ & 1 & 5 & 6 \\
\hline $4-6$ & 15 & 17 & 32 \\
\hline $7+$ & 28 & 14 & 42 \\
\hline Total & 44 & 36 & 80 \\
\hline
\end{tabular}

Among live babies, Apgar of 0-3 were in 6(7.5\%), 4-6 were in $32(40 \%)$ an 7+ were in 42 (52.5\%). Among group with raised LFT Apgar of 0-3 were in 5(13.9\%), 4-6 was in 17 (47.2\%) and $7+$ were in 14 (38.9\%). Statistic study showed significant co-relation between the raised LFT and Apgar score of one minute. Nearly 50\% cases had less than 7 score. $(\mathrm{CC}=0.278 ; \mathrm{P}=$ 0.035 significant).

Table no. 3 Apgar of 5 min and LFT in severe PE:

\begin{tabular}{|l|l|l|l|}
\hline \multirow{2}{*}{ AP5 } & LFT & Total \\
\cline { 2 - 4 } & No & Yes & \\
\hline $0-3$ & & 1 & 1 \\
\hline $4-6$ & 5 & 8 & 13 \\
\hline $7+$ & 39 & 27 & 66 \\
\hline Total & 44 & 36 & 80 \\
\hline
\end{tabular}

Among live babies, Apgar of 0-3 were in 1(1.3\%), 4-6 was in $13(16.3 \%)$ and $7+$ were in 66 (82.5\%). Among group with raised LFT Apgar of 0-3 were in 1(2.8\%), 4-6 was in $8(22.2 \%)$ and $7+$ were in 27 (75\%). Statistic study showed no significant co-relation between the raised LFT and Apgar score of 5 Min. (CC= 0.193; P = 0.212 NS) 
Table no. 4: NICU admission and LFT in severe PE:

\begin{tabular}{|l|l|l|l|}
\hline \multirow{2}{*}{ NICU } & LFT & Total \\
\cline { 2 - 4 } & No & Yes & \\
\hline No & 30 & 18 & 48 \\
\hline Yes & 22 & 30 & 52 \\
\hline Total & 52 & 48 & 100 \\
\hline
\end{tabular}

Out of total cases, 52 babies (52\%) needed ICU admission. Among group with raised LFT 30 babies (62.5\%) needed ICU admission. Statistic study showed significant co-relation between the raised LFT and need of NICU admission. ( $C C=.198 ; \mathrm{P}=0.043$ significant). Similarly among group with raised LFT, $24(50 \%)$ cases had complications. Altered renal function was most common seen in $11(22.1 \%$ ) of cases . 2 (4.2\%) cases had PPH, 6(12.5\%) had abruptio, 3 (6.3\%) cases had pulmonary complications, $3(6.3 \%)$ cases had cardiac complications. $(\mathrm{CC}=0.427 ; \mathrm{P}=$ 0.004 significant).

Table no. 5: Foetal morbidity and LFT in severe PE;

\begin{tabular}{|l|l|l|l|}
\hline \multirow{2}{*}{ FETCOMP } & \multicolumn{2}{l}{ LFT } & \multirow{2}{*}{ Total } \\
\cline { 2 - 4 } & No & Yes & \\
\hline IUGR* & 11 & 12 & 23 \\
\hline Hypoxia & 6 & 8 & 14 \\
\hline IUGR-ARDS** & 6 & 4 & 10 \\
\hline IUGR \& hypoxia & 5 & 10 & 15 \\
\hline Meconium aspiration syndrome(MAS) & 2 & 1 & 3 \\
\hline ARDS & 1 & 2 & 3 \\
\hline Hypoxia-ARDS & 1 & 4 & 5 \\
\hline Total & 32 & 41 & 73 \\
\hline
\end{tabular}

* IUGR- Intrauterine growth retardation

**ARDS- Acute respiratory distress syndrome

Statistic study showed significant co-relation between the raised LFT and foetal complications ( $\mathrm{CC}=0.384 ; \mathrm{P}=0.015$ ). Totally $78(78 \%$ ) cases had foetal complications due to severe PE. Among over all SEP 48(48\%) cases had IUGR, Hypoxia32 (32\%), ARDS 15 (15\%), MAS 10 (10\%). Among group with raised LFT 45 (93.7\%) cases had foetal complications. Among them 26(54.1\%) cases had IUGR, Hypoxia20 (41.6\%), ARDS10 (24.8\%), MAS 7 (14.6\%).

\section{DISCUSSION:}

Liver functions in severe pre-eclampsia:In severe pre-eclampsia there are alterations in the hepatic functions and integrity including delayed excretion of bromosulphthalein and elevation of liver enzymes. Abnormal liver function tests occur in $20 \%$ to $30 \%$ of pregnancies complicated by pre-eclampsia and are associated with poor maternal and fetal outcomes.11,12,14 Various authors have reported alterations in liver enzymes in preeclampsia patients. ${ }^{15-17}$

Age and parity (Graph 1 and 2): Spectrum of pre-eclampsia is more common in extremes, of reproductive age groups. ${ }^{18}$ 
In our study maximum number of severe pre-eclampsia were below 25 yrs (80\%) with mean age of (22.3 age) and associated with increased liver function test were also more common in same age group (81.3\%), which correlates with historical data. Both severe preeclampsia and cases associated with increased liver function test were more common in primigravida $56 \%$ and $58.3 \%$ respectively.

Antenatal check-up (Graph 3): Complications ${ }^{2}$ of severe pre-eclampsia are more common in patients having inadequate antenatal care, which is also supported by low socio economic status and people coming from under developed areas. In our study also complications are more common with un-booked cases. $64.6 \%$ of increased liver function test were of unbooked cases. Even though equal number of booked and un-booked cases seen in the complete study group.

Perinatal outcome (Table-1, 2 and 3): In cases of SEP and HELLP Syndrome neonatal death rates of 6 to 37\% deaths are likely the result of placental insufficiency and hypoxia as suggested by Reubinoff. 19

In study of Nagoya ${ }^{2}$ suggests an increased mortality and morbidity in new-borns of mothers complicated with HELLP syndrome that can be partly attributed to increased rates of intrauterine growth restriction and fetal distress, particularly beyond 32 weeks of gestation. The incidence of intrauterine growth restriction $61.2 \%$, intrauterine fetal death $13.9 \%$ and fetal distress $35.4 \%$ in cases of HELLP syndrome.

Perinatal mortality was high in HELLP syndrome, ranging from 79 to 367 per 1000 live Births in study of Dooh. ${ }^{20}$ In our study total birth of live babies were $78 \%$, IUD - $22 \%$, and neonatal deaths $14 \%$. In cases with raised liver function test incidence were $77.3 \%, 22.9 \%$, $16.7 \%$, respectively. Preterm deliveries were $43 \%$ in overall study group and in cases with raised liver function test $51.1 \%$. ( $\mathrm{P}=.078$ not significant).

Need for NICU admission (Table-4): In study group total number of babies needed NICU admission were $52 \%$ and in cases of raised liver function test it was $62.5 \%(\mathrm{CC}=0.198 ; \mathrm{P}=$ 0.043P significant).

Foetal complications (Table-5): (P value .015 significant)

Incidence of foetal complication was 78(78\%) and in cases with increased LFT was 45(93.7\%). Total number of IUGR in study group was $48 \%$, and in cases of raised liver function test it was $54 \%$. Total number of hypoxia in study group was $32 \%$, and in cases of raised liver function test it was $41.6 \%$. Total number of ARDS in study group was $15 \%$, and in cases of raised liver function test it was $21.3 \%$. Total number of MAS in study group was $10 \%$, and in cases of raised liver function test were $14.6 \%$.

CONCLUSION: Detection of increased LFT in cases of SPE is a special risk category, associated with increased rate of feto-maternal complications, compared to SPE with normal LFT. Such cases need special attention with early detection and referral to higher centre with better facilities of ICU, laboratories and NICU set up to reduce the complications and mortality.

\section{REFERENCES:}

1. Thiagarajah S,Bourgeois Fl, Harbert GM,et al :Thrombocytopenia in preeclampsia: Associated abnormalities and management principles. Am J Obstet Gynecol 1984;150:1. 
2. HELLP Syndrome: 7 Years' Experience from one Referral Center in South-Eastern Turke Nagoya Medical Journal 2000; 43:205-214.

3. Liu CM, Cheng PJ, Chang SD. Maternal Complications and Perinatal Outcomes Associated with Gestational Hypertension and SPE in Taiwanese Women. J Formos Med Assoc. 2008;107(2):129-138

4. Raval DS, Co S, Reid MA, Pildes R. Maternal and neonatal outcome of pregnancies complicated with maternal HELLP syndrome. J Perinatol 1997; 17:266-9.

5. Reubinoff BE, Schenker G: HELLP syndrome-a syndrome of hemolysis, elevated liver enzymes and low platelet count-complicating preeclampsia-eclampsia. Int Gynecol Obstet 1991;36:95.

6. Manas KJ, Welsh JO, Rankin RA et al. Hepatic haemorrhage without rupture in preeclampsia. In Engl J Med 1985; 312:424.

7. Mckenna J, Dover NL, Brame RG. Preeclampsia associated with hemolysis, elevated liver enzymes and low platelets - an obstetric emergency. Obstet Gynecol 1983; 62:751.

8. Beller FK, Dame WR, Ebert C. Pregnancy induced hypertension complication by thrombocytopenia, haemolysis and elevated liver enzyme syndrome. Renal biopsies and outcome. Aust NZ J Obstet Gynecol 1985; 25: 83.

9. Sibai BM, Taslimi MM, EI-Nazer A, et al. Maternal perinatal outcome associated with the syndrome of haemolysis, elevated liver enzymes, and low platelets in severe preeclmapsia - eclampsia. Am J Obstet Gynecol 1986; 155: 501.

10. Haemmerli Jaundice during pregnancy withspecialR. Emphasis on recurrent jaundice during pregnancy and its differential diagnosis. Acta Med Scand 1966; 179: 1.

11. Borglin NE. Serum transaminase activity in uncomplicated and complicated pregnancy and in new born. $J$ Clin Endocrin Metab 1958; 18:872-877.

12. Verghaeghe J, Anthony J, Davey Da. Platelet count and liver function tests in proteinuric and chronic hypertension inpregnancy. S. Afr Med J $1990 ; 79: 590-594$.

13. Hibbard LT. Maternal mortality due to acute toxaemia. Obstet Gynecol 1973; 42:263.

14. Seymour CA, Chadwick VS. Liver and gastrointestinal function in pregnancy. Post Graduate Med J 1979; 55:343-352.

15. McMahon LP, O'Coigligh S, Redman CWG. Hepatic enzymes and the HELLP syndrome a long standing errors. Br J Obstet Gynaecol 1999; 100:693-695.

16. Churchill D, Kilby MD, Bignell A et al. Gamma glutamyl transferase activity in gestational hypertension. Br. J. Obstet Gynecol 1994; 101:252-253.

17. Shukl-a P.K., Sharma D, and MandaI RK. - Serum lactate dehydrogenase in detecting liver damage associated with pre eclampsia. Br J Obstet Gynaecol 1978; 85: 40-42.

18. Maureen Ohara Padden, HELLP Syndrome: Recognition and Perinatal Management Naval Hospital, Camp Pendleton Calfornia, 2002.

19. Reubinoff BE, Schenker ]G: HELIP syndrome-a syndrome of hemolysis, elevated liver enzymes and low platelet count-complicating preeclampsia-eclampsia.Int Gynecol Obstet 1991;36:95, 434.

20. Dooh J, Hohmann M, Kyel PG. Neonatal morbidity and mortality associated with maternal haemolysis elevated liver enzymes and low platelets syndrome. Eur J Pediatr 1997; 156:389-91. 